\title{
Evaluación de la inteligencia sensoriomotriz y del desarrollo psicomotor en lactantes clínicamente sanos asistidos en el sector público de salud Assessment of sensorimotor intelligence and psychomotor development in clinically healthy infants assisted in the public health sector
}

\author{
Lic. María F. Romero ${ }^{a}$, Lic. Juan P. Copparoni ${ }^{a}$ Dra. María V. Fasano, \\ Dra. Marisa Sala ${ }^{a}$ Dra. Mariela Mansilla ${ }^{a}$ Dra. Agustina Vericat ${ }^{a} y$ \\ Bqca. Liliana Disalvo ${ }^{a}$
}

\section{RESUMEN}

Introducción: La primera infancia es el período de mayor neuroplasticidad. La detección temprana de alteraciones del desarrollo permite la intervención oportuna para reducir secuelas y/o complicaciones.

Objetivo: Evaluar el desarrollo psicomotor y cognitivo en lactantes asistidos en el sector público de salud, entre los 6 y 9 meses de edad. Población y métodos: Estudio descriptivo de seguimiento, de niños nacidos a término, clínicamente sanos en la evaluación, con peso, talla y perímetro cefálico adecuados para la edad. Se utilizó la Escala de Evaluación del Desarrollo Psicomotor y la Escala Argentina de Inteligencia Sensorio-motriz entre los 6 y 9 meses. En los casos en que se detectaron riesgo o retraso, se intervino con estimulación temprana. Se determinaron las prevalencias de riesgo/retraso del desarrollo psicomotor e inteligencia sensorio-motriz. Se utilizó el paquete estadístico R, versión 3.3.1.

Resultados: Fueron evaluados 102 niños. A los 6 meses, el 22,5\% presentó riesgo / retraso en el desarrollo. Las prevalencias de resultados no esperados para la edad en desarrollo psicomotor $\mathrm{y}$ en inteligencia sensorio motriz fueron del

a. Instituto de Desarrollo e Investigaciones Pediátricas (IDIP), Hospital de Niños Sor María Ludovica, La Plata, Argentina.

Correspondencia:

Lic. María F. Romero: romero.mflor@gmail.com

Financiamiento:

SANCOR Cooperativas

Unidas Limitada

(C.U.L.).

Conflicto de intereses:

Ninguno que declarar.

Recibido: 24-4-2018

Aceptado: 24-1-2019 http:/ / dx.doi.org/ 10.5546/ aap.2019.224

http:/ / dx.doi.org/10.5546/ aap.2019.eng.224
Cómo citar: Romero MF, Copparoni JP, Fasano MV, Sala M,et al. Evaluación de la inteligencia sensoriomotriz y del desarrollo psicomotor en lactantes clínicamente sanos asistidos en el sector público de salud. Arch Argent Pediatr 2019;117(4):224-229.

\section{INTRODUCCIÓN}

La primera infancia es una etapa crítica y vulnerable. Se considera el período posnatal de mayor neuroplasticidad, en el cual la lactancia materna y el vínculo generado por esta práctica tendrán consecuencias inmediatas y mediatas sobre el óptimo desarrollo del niño. ${ }^{1}$

El desarrollo infantil es un proceso dinámico y continuo de organización progresiva de funciones biológicas, psicológicas y socioculturales en compleja interacción. Se da desde la concepción hasta la madurez y está íntimamente relacionado con el desarrollo del sistema nervioso. ${ }^{2}$ La medición de los trastornos del desarrollo es relevante, sobre todo, en un período crítico y vulnerable de 0 a 5 años. ${ }^{3}$

Varias herramientas de diferente complejidad son útiles para evaluar diversos aspectos del neurodesarrollo. Entre ellas, la Escala de Desarrollo Psicomotor (EEDP), instrumento de pesquisa cualicuantitativo del desarrollo en niños de 0 a 24 meses de edad, permite obtener un coeficiente de desarrollo. Es una herramienta que evalúa de forma objetiva, válida y confiable el rendimiento de los lactantes frente a situaciones que, para 
ser resueltas, requieren determinado grado de desarrollo psicomotor. ${ }^{4}$

A través de la Escala Argentina de Inteligencia Sensoriomotriz (EAIS) es posible evaluar la inteligencia sensoriomotriz de bebés de 6 a 24 meses, lo que posibilita la detección de perturbaciones en el área cognoscitiva. Posee valores estándares de referencia de la población argentina. ${ }^{5}$

En nuestra región, son escasas las publicaciones sobre prevalencias de retraso del desarrollo en niños "aparentemente sanos". Schapira y Oiberman plantean que "ambas escalas pueden considerarse complementarias e indispensables, ya que permiten la evaluación integral del niño". 6,7

Si bien hay algunas publicaciones en las que se utilizó una u otra escala, no se han hallado casos en los que se utilizaran ambas herramientas en un mismo estudio, en una población clínicamente sana.

Se considera, para el presente trabajo, que la utilización de ambas herramientas permite evaluar aspectos diferentes del desarrollo del niño, lo que favorece una visión integral de este y posibilita la detección precoz de alteraciones en una o más áreas del desarrollo. Esta mirada favorece la intervención oportuna y la prevención de consecuencias negativas a largo plazo.

El presente estudio tiene como objetivo evaluar el desarrollo psicomotor y la inteligencia sensoriomotriz en los lactantes a los 6 y a los 9 meses de edad asistidos en el sector público de salud.

\section{POBLACIÓN Y MÉTODOS}

Se evaluaron lactantes a los 6 y a los 9 meses de vida que asistían a los controles pediátricos del Observatorio de Salud del Instituto de Desarrollo e Investigaciones Pediátricas (IDIP), Hospital Sor María Ludovica, durante el período marzodiciembre de 2011.

\section{Diseño}

Se realizó un estudio observacional descriptivo, de seguimiento. Se incluyeron niños de 6 meses de edad, nacidos a término (entre las semanas 37 y 41 de gestación), con peso, talla y perímetro cefálico adecuados para la edad y cuyos padres habían aceptado participar del estudio y habían firmado el consentimiento informado (CI).

Fueron excluidos los niños con enfermedad en curso, síndromes genéticos o patología crónica, que hubieran padecido alguna afección del sistema nervioso central (SNC) y los niños de bajo peso al nacer.

El muestreo fue intencional. Se incluyeron todos los niños que cumplían con los criterios de inclusión y fueron atendidos entre los meses de marzo y diciembre de 2011. Presumiendo una prevalencia de trastornos en el desarrollo del $20 \% 8,9$, el tamaño muestral fue calculado para estimar la prevalencia de trastornos en el desarrollo en los niños de 6 y 9 meses, con una confianza del $95 \%$ y un error de 0,07. El número estimado fue de 126 niños, que fue, finalmente, ajustado por una posible pérdida en el seguimiento del $15 \%$ y llegó, entonces, a ser 145 el número de niños para ser enrolados.

Se evaluó el desarrollo psicomotor, el cual se definió como la progresiva y ordenada adquisición de habilidades funcionales del niño durante el crecimiento, a través de la EEDP, que evaluaba las áreas social, lenguaje, coordinación y motricidad. ${ }^{4-10}$

Esta prueba arrojaba coeficientes de desarrollo (CD) que se clasificaban en las siguientes categorías:

Mayor de 0,85 o igual: normal.

Entre 0,84 y 0,7: riesgo.

Menor de 0,69 o igual: retraso.

La inteligencia sensoriomotriz, definida por J. Piaget como la capacidad de resolver problemas a partir de actividades en las que intervenían, sobre todo, la percepción, las actitudes (el tono) y los movimientos sin evocaciones simbólicas (antes de la aparición del lenguaje), ${ }_{1}^{11}$ fue evaluada a través de la EAIS, que arrojaba las siguientes categorías:

Percentil de 50 a 100: normal.

Percentil mayor de 10 y menor de 50: riesgo.

Percentil 10 o menos: retraso.

Además, se relevaron los datos sociodemográficos edad, escolaridad, situación laboral de los padres y antecedentes obstétricos.

\section{Consideraciones éticas}

El estudio fue aprobado por el Comité Institucional de Revisión de Protocolos de Investigación (CIRPI) del Hospital de Niños Sor María Ludovica en marzo de 2011.

\section{Análisis estadístico}

Los datos obtenidos se analizaron con el paquete estadístico $R$, versión 3.3.1. Se determinaron las prevalencias de riesgo o retraso del desarrollo psicomotor y de la inteligencia 
sensoriomotriz. Las variables cualitativas se informaron como porcentajes (frecuencias), y las cuantitativas, como mediana (rango intercuartil -interquartile range; IQR, por sus siglas en inglés-) Las prevalencias de resultados no esperados para la edad en ambas escalas se informaron con su respectivo intervalo de confianza (IC) del $95 \%$.

\section{RESULTADOS}

Inicialmente, fueron incorporados al estudio 140 niños de 6 meses de edad; sin embargo, 38 niños, por distintos motivos, discontinuaron el seguimiento, por lo cual la muestra final estuvo conformada por 102 niños. Todas las familias a las que se invitó a participar del protocolo aceptaron formar parte.

Los niños incorporados nacieron de un embarazo sin complicaciones y a término. El $8 \%$ (8) de los padres se encontraban desocupados y el $71 \%$ (72) presentaban empleo informal. El $70 \%$ (71) de las madres eran amas de casa. En la Tabla 1, se presentan las características sociodemográficas generales.

A los 6 meses, el 22,5\% (23) de los lactantes presentaron riesgo o retraso en el desarrollo. Las prevalencias de resultados no esperados para la edad, según la EEDP y la EAIS, fueron

TABla 1. Caracterización de la muestra. N: 102

\begin{tabular}{lc}
\hline Variable & $\begin{array}{c}\text { Mediana (IQR) } \\
\text { N (\%) }\end{array}$ \\
\hline Sexo (F) & $54(53)$ \\
Edad gestacional (semanas) & 39 \\
& $(38-40)$ \\
Peso de nacimiento (g) & 3300 \\
& $(2500-4000)$ \\
Perímetro cefálico a los 6 meses (cm) & 42 \\
& $(39-48)$ \\
Peso a los 6 meses (g) & 7620 \\
& $(5400-10970)$ \\
Talla a los 6 meses (cm) & 65 \\
& $(51-89)$ \\
Edad materna (años) & $24(21-30)$ \\
Edad paterna (años) & $29(23-34)$ \\
Escolaridad materna (secundario incompleto) & $65(64)$ \\
Escolaridad paterna (secundario incompleto) & $70(69)$ \\
Lactancia materna exclusiva & \\
a los 6 meses de vida & $44(43)$ \\
Tipo de parto (vaginal) & $72(71)$ \\
Colecho & $48(47)$ \\
\hline
\end{tabular}

IQR: rango intercuartílico. del $13,7 \%$ (el 7,0 \%; el 20,4\%) y del $16,7 \%$ (el 9,4\%; el 23,9\%), respectivamente. De los niños que puntuaron mal, el 34,7 \% (8/23) fallaron en ambas pruebas.

Independientemente de los resultados globales de la EEDP, a los 6 meses de vida, el área del lenguaje fue la más comprometida (Tabla 2 ).

De los 23 lactantes que asistieron a estimulación y fueron reevaluados a los 9 meses de edad, el $84 \%$ (19) de los niños mostraron resultados normales en la EEDP en la segunda evaluación y el $16 \%$ (4), en riesgo; ninguno en retraso. Del mismo modo, el $79 \%$ (18) fueron normales según la EAIS en la segunda evaluación y el $21 \%$ (5) presentaron riesgo; ninguno resultó en retraso.

A los 9 meses, la prevalencia de riesgo/ retraso fue del 20,6\%, que fue del $14,7 \%$ (el 7,8 \%; el $21,6 \%$ ) en la EEDP y del 9,8\% (el 4,0 \%; el 15,6\%) en la EAIS.

El $88 \%$ de los lactantes que puntuaron normal en la primera evaluación en el desarrollo psicomotor volvieron a presentar resultados acordes a lo esperado al ser reevaluados. En tanto que un $12 \%$ pasaron a puntuar en riesgo. Respecto de aquellos lactantes que, a los 6 meses, presentaron resultados en riesgo, el $77 \%$, al ser reevaluados, puntuaron dentro de lo esperado para su edad cronológica; el $23 \%$ permanecieron en riesgo, y el único niño que, en la primera evaluación, mostraba retraso en EEDP pasó a riesgo en la segunda (Tabla 3 ).

E1 $94 \%$ de los lactantes que puntuaron "normal" en inteligencia sensoriomotriz, a los 6 meses de vida, volvieron a presentar resultados acordes a lo esperado a los 9 meses, mientras que un $6 \%$ de los niños que, a los 6 meses, presentaron resultados acordes a la edad en la EAIS presentaron riesgo (el $4 \%$ ) o retraso (el $2 \%)$ al ser reevaluados. Estos lactantes no recibieron intervención con estimulación temprana entre la primera y la segunda evaluación (Tabla 3).

TABla 2. Resultados de la Escala de Evaluación del Desarrollo Psicomotor por áreas a los 6 meses de edad. N: 102

\begin{tabular}{lc}
\hline Áreas & $\begin{array}{c}\text { No normal } \\
\text { Porcentaje y número de casos }\end{array}$ \\
\hline Lenguaje & $13 \%(13)$, IC $95 \%: 6,3 \% ; 19,2 \%$ \\
Motora & $6 \%(6)$, IC $95 \%: 1,3 \% ; 10,4 \%$ \\
Coordinación & $5 \%(5)$, IC $95 \%: 0,7 \% ; 9,1 \%$ \\
Social & $5 \%(5)$, IC $95 \%: 0,7 \% ; 9,1 \%$ \\
\hline
\end{tabular}

IC: intervalo de confianza. 
Hubo un $21 \%$ (21) de niños que fueron derivados a estimulación temprana en la segunda evaluación, el $81 \%$ (17) de los cuales presentaban resultados "normales" en la primera evaluación.

\section{DISCUSIÓN}

El hallazgo más importante de este estudio es que la observación global del desarrollo de la muestra a los 6 meses de vida de lactantes clínicamente sanos, que concurren al control de salud, evaluada por las dos pruebas (EEDP y EAIS), mostró que el 22,5\% $(n=23)$ de los lactantes presentó indicadores de alteración del desarrollo psicomotor o en la inteligencia sensoriomotriz. De ellos, 17 lactantes presentaron retraso, 8 de los cuales fallaron en ambas pruebas.

A diferencia de Bermúdez y Carabajal, ${ }^{8}$ que, utilizando la EEDP, encontraron un 22,8 \% de riesgo y retraso en el grupo etario de 7 a 12 meses, en nuestros lactantes, se observó un $14,7 \%$ con riesgo o retraso. En el mencionado estudio, evaluaron 559 niños de entre 0 y 24 meses de edad, y realizaron una comparación por semestre, ambas poblaciones correspondieron a muestras tomadas de familias asistidas en el sistema público de salud. Lejarraga et al., ${ }^{9}$ utilizando la Prueba Nacional de Pesquisa (PRUNAPE), encontraron un $20 \%$ de retraso en el desarrollo; los autores evaluaron una población de niños de 0 a 6 años, de diferentes niveles socioeconómicos. Al analizar el grupo de niños de entre 12 y 24 meses, de nivel socioeconómico bajo, dichos autores encontraron resultados similares a los de Bermúdez y Carabajal. ${ }^{8}$

Tal como reflejan los resultados, en nuestro estudio, se observa una menor prevalencia de riesgo o retraso. Si bien resulta aventurado formular una conjetura, se debe considerar que los lactantes de nuestro estudio pertenecen a una población que tiene acceso a los servicios de salud y tiene incorporadas pautas de conducta sanitarias; los niños son llevados periódicamente al control pediátrico y cumplen con el esquema de vacunación. Además, también se debería tener en cuenta la menor sensibilidad por parte de las herramientas de pesquisa a la hora de evaluar a niños de esta edad.

En el presente estudio, el 13,7\% de los lactantes a los 6 meses presentaba riesgo/retraso según la EAIS. Oiberman et al. ${ }^{7-12}$ encontraron, en la población argentina, una prevalencia de riesgo o retraso con la EAIS del 27,75\% (el 14\% de riesgo y el 13,75\% de retraso), la cual, a priori, fue superior a la hallada en nuestra muestra y que podría explicarse por el tamaño muestral de nuestro estudio. Sin embargo, los resultados de Oiberman et al. no fueron separados por edad y no se especificaron las prevalencias para los 6 meses de edad. Además, su muestra fue de niños de 6 a 30 meses, pero incluyó un escaso número de lactantes de 6 y 9 meses (34 lactantes de 6 meses y 36 de 9 meses).

Debe tenerse en cuenta que nuestros hallazgos de prevalencia de riesgo o retraso a los 9 meses no son comparables con los resultados de Bermúdez ni de Oiberman, dado que, en nuestro estudio, los lactantes con riesgo o retraso a los 6 meses fueron intervenidos. Del mismo modo, tampoco se pueden comparar con un estudio más reciente realizado en Chile, que evaluaba, con la EEDP, a niños de 8 a 24 meses de edad, de nivel socioeconómico bajo, en el que hallaron un $28,9 \%$ de niños con riesgo y retraso. ${ }^{13}$

Publicaciones internacionales, como la realizada por Q. Wei et al., ${ }^{14}$ encontraron que el $39,7 \%$ de los niños menores de 3 años evaluados presentaba retraso en el desarrollo en, al menos, una de las cinco áreas. Concluyeron que existía una alta prevalencia de retraso en el desarrollo en los niños menores de tres años en las zonas

TABla 3. Resultados de la Escala de Evaluación del Desarrollo Psicomotor y Escala Argentina de Inteligencia Sensoriomotriz a los 9 meses de vida comparada con los resultados obtenidos a los 6 meses. N: 102

\begin{tabular}{|c|c|c|c|c|}
\hline & & $\begin{array}{c}\text { Normal } \\
\text { N }(\%)\end{array}$ & $\begin{array}{c}\text { da evalt } \\
\text { Riesgo } \\
\text { N }(\%) \\
\end{array}$ & $\begin{array}{c}\text { Retraso } \\
\text { N }(\%)\end{array}$ \\
\hline \multirow[t]{3}{*}{ EEDP, primera evaluación } & Normal & $77(88)$ & $11(12)$ & 0 \\
\hline & Riesgo & $10(77)$ & $3(23)$ & 0 \\
\hline & Retraso & 0 & $1(100)$ & 0 \\
\hline \multirow[t]{2}{*}{ EAIS, primera evaluación } & Normal & $80(94)$ & $3(4)$ & $2(2)$ \\
\hline & Retraso & $12(71)$ & $5(29)$ & 0 \\
\hline
\end{tabular}


afectadas por la pobreza en China y propusieron implementar un programa de detección y atención temprana de esta problemática.

Una debilidad de nuestro estudio se relaciona con la homogeneidad de la muestra en cuanto a las características socioeconómicas, lo que no permitió encontrar relaciones significativas entre el desarrollo psicomotor o la inteligencia sensoriomotriz y el nivel educativo de los padres, la edad de la madre, etc. Tampoco fueron evaluados otros factores, como la presencia de anemia y la deficiencia de micronutrientes, en particular, hierro, cuyo impacto negativo sobre el desarrollo de habilidades psicomotoras ha sido ampliamente documentado. ${ }^{15,16}$

E1 $83 \%$ de los niños que asistieron a estimulación temprana a los 6 meses de vida presentaron resultados acordes a lo esperado para su edad cronológica, en tanto que el $17 \%$ restante, a pesar de presentar resultados no esperados para la edad, mejoró el rendimiento en las pruebas. Aunque hubo una tendencia de evolución favorable luego de la intervención con estimulación temprana, no constituyó evidencia científica sobre la utilidad de este tipo de intervención. Serán necesarias futuras investigaciones que indaguen sobre el tema.

En la revisión internacional, una investigación realizada por Walker et al. demuestra los beneficios de la estimulación psicosocial para favorecer el desarrollo en los niños desnutridos. ${ }^{17}$ En este estudio, los niños que fueron intervenidos durante 2 años con estimulación temprana mejoraron su rendimiento. A su vez, Moreno Mora et al. muestran la eficacia de un programa de estimulación integral realizado a niños de bajo peso con retraso en el desarrollo, que evidencia la tendencia a una evolución favorable. ${ }^{18}$

Como el desarrollo es un proceso dinámico de adquisición de diferentes habilidades de complejidad creciente, los aspectos que se evalúan a los 9 meses no son los mismos que fueron evaluados previamente. Es decir que aquel niño que, a los 6 meses, había puntuado bien en las escalas utilizadas $y$, a los 9 meses, presentaba resultados no acordes a lo esperado no ha podido construir nuevas habilidades en etapas posteriores a las evaluadas.

Dado que, a los 6 y a los 9 meses de edad, un número considerable de lactantes clínicamente sanos fueron derivados a estimulación temprana, siendo esta una población que no era evaluada en su desarrollo en los controles de rutina, se considera importante implementar acciones tendientes a la detección de dificultades en el desarrollo psicomotor e inteligencia sensoriomotriz, que brinde una mirada integral del niño y permita detectar, de manera temprana, indicios o signos de alteración en estas.

\section{CONCLUSIONES}

En esta población, el 22,5\% de los lactantes clínicamente sanos presentó riesgo/ retraso del desarrollo psicomotor y cognitivo a los 6 meses en, al menos, una de las pruebas. La prevalencia a los 9 meses fue mayor que la esperada para esa edad.

La evaluación temprana del desarrollo de los lactantes permitió detectar alteraciones en este e intervenir precozmente.

\section{REFERENCIAS}

1. Cardoso MA, Hure AJ. Nutrition in the first 500 days of life. Public Health Nutr. 2014; 17(9):1907-8.

2. Cafiero PJ. Desarrollo Infantil y sus alteraciones. En Sociedad Argentina de Pediatría. Pronap. 2008;1:62-3.

3. Lejarraga H, Kelmansky D, Passcucci M, Masautis A, et. al. Evaluación del desarrollo psicomotor del niñoen grupos de población como indicador positivo de salud. Arch Argent Pediatr. 2016;114(1):23-9.

4. Rodríguez S, Arancibia V, Undurraga C. Escala de evaluación del desarrollo psicomotor: 0 a 24 meses. 12.a ed. Santiago de Chile: Galdoc; 1996.

5. Oiberman A, Mansilla M, Orellana L. Métodos y resultados. En Nacer y Pensar Construcción de la Escala Argentina de la Inteligencia sensorio Motriz (EAIS) de 6 meses a 2 años. Vol I. Buenos Aires: CIIPME-CONICET; 2002:20-5.

6. Schapira IT. Comentarios y aportes sobre desarrollo e inteligencia sensorio-motriz en lactantes. Análisis de herramientas de evaluación de uso frecuente. Actualización bibliográfica. Rev Hosp Mater Infant Ramón Sardá. 2007;26(1):21-7.

7. Oiberman A, Mansilla M, Orellana L. Evaluación de la inteligencia en bebés argentinos: Escala Argentina de Inteligencia Sensoriomotriz. Arch Argent Pediatr. 2006;104(4):316-23.

8. Bermúdez E, Carabajal N. Evaluación del Desarrollo Psicomotriz en niños de 0 a 24 meses. Arch Argent Pediatr. 1995;93(6):354-62.

9. Lejarraga H, Menéndez A, Menzano E, Guerra L, et al. Screening for developmental problems at primary care level: a field programme in San Isidro, Argentina. Paediatr Perinat Epidemiol. 2008;22(2):180-7.

10. Lejarraga H. Desarrollo del niño en contexto. Buenos Aires: Paidós; 2006.

11. PiagetJ. El nacimiento de la inteligencia en el niño. Ginebra: Neuschatel Delachaux et Niestlé; 1973.

12. Oiberman A, Paolini C, Mansilla M. Escala Argentina de Inteligencia Sensorio Motriz. Percentiles Nacionales. Interdisciplinaria. 2012;29(2):305-23.

13. Santelices Álvarez M, Besoain C, Escobar M. Monoparentalidad, trabajo materno y desarrollo psicomotor infantil: Un estudio chileno en niños que asisten a salas cuna en contexto de pobreza. Univ Psychol. 2015;14(2):675-84.

14. WeiQ, Zhang J,Scherpbier R, Zhao C, et al. High prevalence of developmental delay among children under three years 
of age in poverty-stricken areas of China. Public Health. 2015;129(12):1610-7.

15. Carter RC, Jacobson JL, Burden MJ, Armony-Sivan R, et al. Iron deficiency anemia and cognitive function in infancy. Pediatrics. 2010;126(2):e427-34.

16. Lozoff B, Smith J, Kaciroti N, Clark K, et al. Functional significance of early-life iron deficiency: outcomes at 25 years. J Pediatr. 2013;163(5):1260-6.

17. Walker SP, Chang S, Powell CA, Baker-Henningham
H.Building human capacity through early childhood intervention: the Child Development Research programme at the Tropical Medicine Research Institute, The University of the West Indies, Kingston, Jamaica. West Indian Med J. 2012;61(4):316-22.

18. MorenoMora R,Pérez DíazC. Retardoenel neurodesarrollo en niños de la Habana Vieja". Rev Cubana Med Gen Integr. 2008;24(3) [Consulta: 28 de enero de 2019]. Disponible en: http:/ / scielo.sld.cu/pdf/mgi/v24n3/mgi12308.pdf.

\section{Artículos seleccionados}

Los siguientes resúmenes y comentarios de trabajos seleccionados se encuentran disponibles en la versión electrónica de este número.

Pediatrics. 2019 Feb;143(2)

Hallazgos retinianos en niños pequeños con aumento de la presión intracraneana de causas no traumáticas (Shi A, et al. Retinal finding in young children with increased intracranial pressure from non traumatic causes)

Comentario: Dr. Javier Indart de Arza. Unidad de Violencia Familiar. Hospital General de Niños Dr. Pedro de Elizalde. Ciudad de Buenos Aires.

JAMA Pediatr. 2019 Jan 28. doi: 10.1001/jamapediatrics.2018.5056

Asociación entre el tiempo de pantalla y el desempeño de los niños en una prueba de pesquisa de neurodesarrollo (Madigan S, et al. Association between screen time and children's performance on a developmental screening test)

Comentario: Dra. Paula Otero. Subcomisión de TIC de la Sociedad Argentina de Pediatría.

Pediatrics. 2019 Mar;143(3). pii: e20182507

Factores asociados con pasajeros infantojuveniles sin sujetar en choques de vehículos automotores (Roehler DR, et al. Factors associated with unrestrained young passengers in motor vehicle crashes)

Comentario: Dra. Ingrid Waisman. Comité Nacional de Prevención de Lesiones de la Sociedad Argentina de Pediatría.

J Depress Anxiety 2018,7:1

El impacto del uso de dispositivos electrónicos en niños (Sundus M. The impact of using gadgets on children)

Comentario: Dr. Fernando Lamas. Subcomisión de Tecnologías de Información y Comunicación de la Sociedad Argentina de Pediatría.

Pediatrics. 2019 Jan;143(1). pii: e20183348

Eligiendo el juguete adecuado para los niños en la era digital (Healey $A$, et al. Selecting appropriate toys for young children in the digital era)

Comentario: Dr. Fernando Lamas. Subcomisión de Tecnologías de Información y Comunicación de la Sociedad Argentina de Pediatría.

Pediatrics. 2018 Dec;142 (6). pii: e20182119

Tener sobrepeso u obesidad y el desarrollo del asma (Lang JE, et al. Being overweight or obese and the development of asthma)

Comentario: Dra. Verónica Giubergia. Hospital de Pediatria Juan P. Garrahan. Buenos Aires. 